\title{
Remarks on Duffin-Kemmer-Petiau theory and gauge invariance
}

\author{
J. T. Lunardif, B. M. Pimentel, R. G. Teixeira and J. S. Valverde \\ Instituto de Física Teórica \\ Universidade Estadual Paulista \\ Rua Pamplona 145 \\ 01405-900 - São Paulo, S.P. \\ Brazil
}

\begin{abstract}
Two problems relative to the electromagnetic coupling of Duffin-Kemmer-Petiau (DKP) theory are discussed: the presence of an anomalous term in the Hamiltonian form of the theory and the apparent difference between the Interaction terms in DKP and Klein-Gordon (KG) Lagrangians. For this, we first discuss the behavior of DKP field and its physical components under gauge transformations. From this analysis, we can show that these problems simply do not exist if one correctly analyses the physical components of DKP field.
\end{abstract}

\footnotetext{
${ }^{*}$ On leave from Departamento de Matemática e Estatística. Setor de Ciências Exatas e Naturais. Universidade Estadual de Ponta Grossa. Ponta Grossa, PR - Brazil.
} 


\section{Introduction}

The Duffin-Kemmer-Petiau (DKP) equation is a first order relativistic wave equation for spin 0 and 1 bosons [1, 2, 3], similar to Dirac equation. The historical development of this theory, among others, until the 70's can be found in reference [4]. More recently there have been an increasing interest in DKP theory, specifically it has been applied to QCD (large and short distances) by Gribov [5], to covariant Hamiltonian dinamics by Kanatchikov [6] and have been generalized to curved space-time by Red'kov [0] and Lunardi et al [8].

It is well known that for free fields there is a perfect equivalence between DKP equation and Klein-Gordon (KG) and Proca equations but, when interaction with electromagnetic field through minimal coupling is present, doubts about this equivalence arise. This is because when one pass to the Hamiltonian form (Schrödinger like) or to a second order KG like wave equation an anomalous term that does not posses a clear physical interpretation appears [2]. Moreover, it was argued that, when applied to the Lagrangian density for the spin 0 version of DKP theory, the minimal coupling seems to provide an interaction term linear in the potential vector $A^{\mu}$, a different result from the quadratic term in $A^{\mu}$ found when starting with the KG Lagrangian.

Our intention in this paper is to show that the problem in the physical interpretation of DKP theory and the contradiction between it and KG theory are only apparent and can be fully elucidated by a correct interpretation of the physical meaning of the DKP wave function $\psi$ and the correct application of gauge invariance principle.

For this, we will dedicate Section 2 to mention some basic results in DKP free field theory, mainly those relative to the obtainment and interpretation of the physical components of DKP field. For further details we suggest the reader to 
original works [1, 2] or classic textbooks [9, 10]. Next, in Section 3, we analyse the apparent discrepancy between KG and DKP theories when electromagnetic interaction comes to play. This discrepancy is shown to be caused by the use of an expression for the DKP field, in terms of its physical components, that is incompatible with the gauge invariance. Once we construct a correct expression for the DKP field we find that there is a perfect agreement.

In Section 4 we show that the question of the apparently problematic term lacking physical interpretation can be shown to be not relevant by noticing that it disappears when we analyse the physical components of the DKP field $\psi$. This fact has already been shown by Nowakowski [11] at classical level and more recently by Fainberg and Pimentel [12] at classical and quantum level too. But in this paper we adopt a different approach to reduce the wave function $\psi$ to its physical components using the projectors on the spin 0 and spin 1 sectors of DKP theory [9] in order to make more clear, in our opinion, the origin of this physical interpretation. Finally, in Section 5, we make some concluding remarks.

\section{The free field theory}

The DKP equation is given by

$$
\left(i \beta^{\mu} \partial_{\mu}-m\right) \psi=0
$$

where the matrices $\beta^{\mu}$ obey the algebraic relations

$$
\beta^{\mu} \beta^{\nu} \beta^{\rho}+\beta^{\rho} \beta^{\nu} \beta^{\mu}=\beta^{\mu} \eta^{\nu \rho}+\beta^{\rho} \eta^{\nu \mu}
$$

being $\eta^{\mu \nu}$ the metric tensor of Minkowski space-time with signature $(+---)$. The matrices $\beta^{\mu}$ have 2 non trivial irreducible representations: a 5 degree one, corresponding to spin 0 particles, and a 10 degree one, corresponding to spin 1 
particles. From the algebraic relation above we can also obtain (no summation on repeated indexes)

$$
\left(\beta^{\mu}\right)^{3}=\eta^{\mu \mu} \beta^{\mu}
$$

so that we can define the matrices

$$
\eta^{\mu}=2\left(\beta^{\mu}\right)^{2}-\eta^{\mu \mu}(\text { no summation })
$$

that satisfy

$$
\begin{gathered}
\left(\eta^{\mu}\right)^{2}=1, \eta^{\mu} \eta^{\nu}-\eta^{\nu} \eta^{\mu}=0, \\
\eta^{\mu} \beta^{\nu}+\beta^{\nu} \eta^{\mu}=0(\mu \neq \nu), \\
\eta^{\mu \mu} \beta^{\mu}=\eta^{\mu} \beta^{\mu}=\beta^{\mu} \eta^{\mu} \text { (no summation) } .
\end{gathered}
$$

With these results we can write the Lagrangian density for DKP free field as

$$
\mathcal{L}=\frac{i}{2} \bar{\psi} \beta^{\mu} \overleftrightarrow{\partial}_{\mu} \psi-m \bar{\psi} \psi
$$

where $\bar{\psi}$ is defined as

$$
\bar{\psi}=\psi^{\dagger} \eta^{0}
$$

Moreover, we can choose $\beta^{0}$ to be hermitian and $\beta^{i}$ anti-hermitian so that the equation for $\bar{\psi}$ can also be easily obtained by applying hermitian conjugation to equation (囵).

Multiplying the DKP equation by $\partial_{\alpha} \beta^{\alpha} \beta^{\nu}$ from left we can find [2]

$$
\partial^{\nu} \psi=\beta^{\alpha} \beta^{\nu} \partial_{\alpha} \psi
$$

and contracting this result with $\partial_{\nu}$ we have

$$
\square \psi+m^{2} \psi=0
$$


so that each component of $\psi$ satisfies $\mathrm{KG}$ equation, as expected for a relativistic field equation. Summing equation (10) for $\nu=0$ and DKP equation multiplied from left by $-i \beta^{0}$ we get a Schrödinger like Hamiltonian form wave equation

$$
i \partial_{t} \psi=H \psi
$$

where

$$
H=i\left[\beta^{i}, \beta^{0}\right] \partial_{i}+m \beta^{0} .
$$

Under a Lorentz transformation $x^{\prime \mu}=\Lambda_{\nu}^{\mu} x^{\nu}$ we have

$$
\begin{aligned}
& \psi \rightarrow \psi^{\prime}=U(\Lambda) \psi, \\
& U^{-1} \beta^{\mu} U=\Lambda_{\nu}^{\mu} \beta^{\nu},
\end{aligned}
$$

and for infinitesimal transformations $\Lambda^{\mu \nu}=\eta^{\mu \nu}+\omega^{\mu \nu}\left(\omega^{\mu \nu}=-\omega^{\nu \mu}\right)$ we obtain [9]

$$
U=1+\frac{1}{2} \omega^{\mu \nu} S_{\mu \nu}, S_{\mu \nu}=\left[\beta_{\mu}, \beta_{\nu}\right]
$$

The spin 0 and spin 1 sectors of the theory can be selected from a general representation of $\beta^{\mu}$ matrices through a set of operators, as shown in reference [9]. For the spin 0 sector the operators are

$$
P=-\left(\beta^{0}\right)^{2}\left(\beta^{1}\right)^{2}\left(\beta^{2}\right)^{2}\left(\beta^{3}\right)^{2}
$$

which satisfies $P^{2}=P$, and

$$
P^{\mu}=P \beta^{\mu}
$$

It can be shown that

$$
P^{\mu} \beta^{\nu}=P \eta^{\mu \nu}, P S_{\mu \nu}=0
$$

and, as consequence, under infinitesimal Lorentz transformations (16) we have

$$
P U \psi=P \psi,
$$


so that $P \psi$ transforms as a (pseudo)scalar. Similarly

$$
P^{\mu} U \psi=P^{\mu} \psi+\omega^{\mu}{ }_{\nu} P^{\nu} \psi
$$

showing that $P^{\mu} \psi$ transforms like a (pseudo)vector.

Applying these operators to DKP equation (11) we have

$$
\partial_{\nu}\left(P^{\nu} \psi\right)=\frac{m}{i} P \psi
$$

and

$$
P^{\nu} \psi=\frac{i}{m} \partial^{\nu}(P \psi)
$$

which combined provide

$$
\partial^{\mu} \partial_{\mu}(P \psi)+m^{2}(P \psi)=\square(P \psi)+m^{2}(P \psi)=0
$$

These results show that all elements of the column matrix $P \psi$ are scalar fields of mass $m$ obeying KG equation while the elements of $P^{\mu} \psi$ are $\frac{i}{m}$ times the derivative with respect to $x_{\mu}$ of the corresponding elements of $P \psi$. Then, acting $P$ upon $\psi$ selects the spin 0 sector of DKP theory, making explicitly clear that it describes a scalar particle.

Now, for the spin 1 sector we have as operators

$$
R^{\mu}=\left\{\begin{array}{c}
\left(\beta^{1}\right)^{2}\left(\beta^{2}\right)^{2}\left(\beta^{3}\right)^{2} \beta^{\mu} \beta^{0} ; \mu \neq 0 \\
-\left(\beta^{1}\right)^{2}\left(\beta^{2}\right)^{2}\left(\beta^{3}\right)^{2}\left(1-\left(\beta^{0}\right)^{2}\right) ; \mu=0
\end{array}\right.
$$

which can be written compactly as

$$
R^{\mu}=\left(\beta^{1}\right)^{2}\left(\beta^{2}\right)^{2}\left(\beta^{3}\right)^{2}\left[\beta^{\mu} \beta^{0}-\eta^{\mu 0}\right]
$$

and

$$
R^{\mu \nu}=R^{\mu} \beta^{\nu}
$$


From these definitions we have the following properties

$$
\begin{gathered}
R^{\mu \nu}=-R^{\nu \mu}, \\
R^{\mu} \beta^{\nu} \beta^{\alpha}=\eta^{\nu \alpha} R^{\mu}-\eta^{\mu \alpha} R^{\nu}, \\
R^{\mu} S^{\nu \alpha}=\eta^{\mu \nu} R^{\alpha}-\eta^{\mu \alpha} R^{\nu}, \\
R^{\mu \nu} S^{\alpha \beta}=\eta^{\nu \alpha} R^{\mu \beta}-\eta^{\mu \alpha} R^{\nu \beta}-\eta^{\nu \beta} R^{\mu \alpha}+\eta^{\mu \beta} R^{\nu \alpha} .
\end{gathered}
$$

These results allow us to show that under infinitesimal Lorentz transformations we have

$$
R^{\mu} U \psi=R^{\mu} \psi+\omega^{\mu}{ }_{\alpha} R^{\alpha} \psi
$$

so we can see that $R^{\mu} \psi$ transforms like a (pseudo)vector while $R^{\mu \nu} \psi$ transforms like a (pseudo)tensor since

$$
R^{\mu \nu} U \psi=R^{\mu \nu} \psi+\omega_{\beta}^{\nu} R^{\mu \beta} \psi+\omega_{\alpha}^{\mu} R^{\alpha \nu} \psi
$$

The application of these operators to DKP equation results in

$$
\partial_{\nu}\left(R^{\mu \nu} \psi\right)=\frac{m}{i} R^{\mu} \psi
$$

and

$$
R^{\mu \alpha} \psi=-\frac{i}{m} U^{\mu \alpha}
$$

where

$$
U^{\mu \alpha}=\partial^{\mu} R^{\alpha} \psi-\partial^{\alpha} R^{\mu} \psi
$$

is the strength tensor of the massive vector field $R^{\mu} \psi$. Combined, these results provide

$$
\begin{gathered}
\partial_{\nu}\left(-\frac{i}{m} U^{\mu \nu}\right)=\frac{m}{i} R^{\mu} \psi \\
\partial_{\nu} U^{\nu \mu}+m^{2} R^{\mu} \psi=0,
\end{gathered}
$$


or equivalently

$$
\left(\square+m^{2}\right) R^{\mu} \psi=0 ; \partial_{\mu} R^{\mu} \psi=0 .
$$

So, all elements of the column matrix $R^{\mu} \psi$ are components vector fields of mass $m$ obeying Proca equation; being the elements of $R^{\mu \alpha} \psi$ equal to $\frac{-i}{m}$ times the field strength tensor of the vector field of which the corresponding elements of $R^{\mu} \psi$ are components. So, similarly to the spin 0 case, this procedure selects the spin 1 content of DKP theory, making explicitly clear that it describes a massive vectorial particle.

Moreover, for the spin 0 case, we can choose a 5 degree irreducible representation of the $\beta^{\mu}$ matrices in such a way that

$$
P \psi=P\left(\begin{array}{c}
\psi_{0} \\
\psi_{1} \\
\psi_{2} \\
\psi_{3} \\
\psi_{4}
\end{array}\right)=\left(\begin{array}{c}
0 \\
0 \\
0 \\
0 \\
\psi_{4}
\end{array}\right) ; P_{\mu} \psi=\left(\begin{array}{c}
0 \\
0 \\
0 \\
0 \\
\psi_{\mu}
\end{array}\right)(\mu=0, \ldots, 3)
$$

This representation is

$$
\begin{aligned}
& \beta^{0}=\left(\begin{array}{ccccc}
0 & 0 & 0 & 0 & 1 \\
0 & 0 & 0 & 0 & 0 \\
0 & 0 & 0 & 0 & 0 \\
0 & 0 & 0 & 0 & 0 \\
1 & 0 & 0 & 0 & 0
\end{array}\right) ; \beta^{1}=\left(\begin{array}{ccccc}
0 & 0 & 0 & 0 & 0 \\
0 & 0 & 0 & 0 & 1 \\
0 & 0 & 0 & 0 & 0 \\
0 & 0 & 0 & 0 & 0 \\
0 & -1 & 0 & 0 & 0
\end{array}\right) ; \\
& \beta^{2}=\left(\begin{array}{ccccc}
0 & 0 & 0 & 0 & 0 \\
0 & 0 & 0 & 0 & 0 \\
0 & 0 & 0 & 0 & 1 \\
0 & 0 & 0 & 0 & 0 \\
0 & 0 & -1 & 0 & 0
\end{array}\right) ; \beta^{3}=\left(\begin{array}{ccccc}
0 & 0 & 0 & 0 & 0 \\
0 & 0 & 0 & 0 & 0 \\
0 & 0 & 0 & 0 & 0 \\
0 & 0 & 0 & 0 & 1 \\
0 & 0 & 0 & -1 & 0
\end{array}\right) .
\end{aligned}
$$


From now on we will use this specific representation. So, equation (23) and (40) will result in (for $\nu=0,1,2,3$ )

$$
\psi_{\nu}=\frac{i}{m} \partial^{\nu}\left(\psi_{4}\right)
$$

We can now make

$$
\psi_{4}=\sqrt{m} \varphi
$$

where $\varphi$ is a scalar field, obtaining

$$
\psi_{\nu}=\frac{i}{\sqrt{m}} \partial_{\nu} \varphi
$$

so that

$$
\psi=\left(\begin{array}{c}
\frac{i}{\sqrt{m}} \partial_{\nu} \varphi \\
\sqrt{m} \varphi
\end{array}\right)
$$

and consequently

$$
P \psi=\left(\begin{array}{c}
0_{4 \times 1} \\
\sqrt{m} \varphi
\end{array}\right), P^{\mu} \psi=\frac{i}{\sqrt{m}}\left(\begin{array}{c}
0_{4 \times 1} \\
\partial^{\mu} \varphi
\end{array}\right), \square \varphi+m^{2} \varphi=0 .
$$

Here we will call expression (46) for $\psi$ the physical form of DKP field. If we use this representation for the $\beta$ matrices, together with the physical form of $\psi$, in DKP Lagrangian (8) we get

$$
\mathcal{L}=-\frac{1}{2}\left(\varphi^{*} \square \varphi+\varphi \square \varphi^{*}\right)-m^{2} \varphi^{*} \varphi
$$

which can be integrated by parts giving as result, except by a four-divergence term, the usual KG Lagrangian

$$
\mathcal{L}=\partial^{\mu} \varphi^{*} \partial_{\mu} \varphi-m^{2} \varphi^{*} \varphi
$$

Similarly, for the case of spin 1 particles, a 10 degree irreducible representation of the matrices can be found such that the first four components of the field $\psi$ 
are the components of a four vector $B^{\mu}$, being selected by $R^{\mu}$. The other 6 components of $\psi$ are the 6 independent components of the strength tensor of $B^{\mu}$. These components are selected by $R^{\mu \nu}$ so that when we apply these operators to equation (38) it becomes the Proca equation for the field $B^{\mu}$. Anyway, we will not make use of this specific representation so we just mention this possibility here for the sake of completness without working out the complete calculations.

\section{Local gauge invariance and the interaction La- grangian}

When one interacts DKP field with electromagnetic field, a close attention must be taken when interpreting the physical content of the theory. After performing the minimal coupling to the DKP Lagrangian we obtain as an interaction term

$$
\mathcal{L}_{I}=e A_{\mu} \bar{\psi} \beta^{\mu} \psi
$$

so that, to regain the interaction term in KG theory, one may be tempted to use the expression (46) for $\psi$ in the interaction term above, getting as result

$$
\mathcal{L}_{I}=i e A^{\mu}\left(\varphi^{*} \partial_{\mu} \varphi-\partial_{\mu}\left(\varphi^{*}\right) \varphi\right)
$$

that is, a linear term different from the quadratic term obtained from KG Lagrangian. This result gave rise to the interpretation that DKP and KG theories were equivalent only in the free field case. Our intention here is to show that this difference arises from the incorrect use of the physical form of DKP field (46) in a situation where it is no longer valid, since it was obtained for free fields, which is not the case anymore.

First of all, we can easily see that expression (46) for the physical form is incompatible with gauge invariance. This comes from the fact that, under local 
gauge transformations, we must have

$$
\psi \rightarrow \psi^{\prime}=e^{i e \alpha(x)} \psi ; \varphi \rightarrow \varphi^{\prime}=e^{i e \alpha(x)} \varphi
$$

for DKP and KG fields, respectively. But using the transformation property of $\varphi$ in the expression (46) for the physical form results in

$$
\psi^{\prime}=\left(\begin{array}{c}
\frac{i}{\sqrt{m}} \partial_{\nu} \varphi^{\prime} \\
\sqrt{m} \varphi^{\prime}
\end{array}\right)=\left(\begin{array}{c}
\frac{i}{\sqrt{m}}\left(i e e^{i e \alpha(x)} \varphi \partial_{\nu} \alpha(x)+e^{i e \alpha(x)} \partial_{\nu} \varphi\right) \\
e^{i e \alpha(x)} \sqrt{m} \varphi
\end{array}\right) \neq e^{i e \alpha(x)} \psi
$$

so that it becomes obvious that the expression obtained in the free field case is no longer valid.

The solution to this problem would be simply change the physical form of $\psi$ from that given by equation (46) to

$$
\psi=\left(\begin{array}{c}
\frac{i}{\sqrt{m}} D_{\nu} \varphi \\
\sqrt{m} \varphi
\end{array}\right)
$$

where $D_{\mu}=\partial_{\mu}-i e A_{\mu}$, which obviously transforms as necessary to keep the compatibility between both transformations in equation (52). More than this, we can easily show that this change in the expression for the physical form arises naturally when we analyse DKP Lagrangian with minimal coupling, showing explicitly that the direct application of equation (46) in this case is a mistake. Indeed, starting with the DKP Lagrangian (8) we perform the minimal coupling obtaining as result

$$
\mathcal{L}=\frac{i}{2}\left(\bar{\psi} \beta^{\mu} D_{\mu} \psi-D_{\mu}^{*}(\bar{\psi}) \beta^{\mu} \psi\right)-m \bar{\psi} \psi
$$

or

$$
\mathcal{L}=\frac{i}{2} \bar{\psi} \beta^{\mu} \overleftrightarrow{\partial}_{\mu} \psi-m \bar{\psi} \psi+e \bar{\psi} A_{\mu} \beta^{\mu} \psi
$$

and from these we obtain the minimally coupled DKP equation 


$$
\left(i \beta^{\mu} D_{\mu}-m\right) \psi=0
$$

Applying the operators $P$ and $P^{\nu}$ we obtain

$$
D_{\nu}\left(P^{\nu} \psi\right)=\frac{m}{i} P \psi
$$

and

$$
P^{\nu} \psi=\frac{i}{m} D^{\nu}(P \psi)
$$

so that

$$
D_{\nu} D^{\nu}(P \psi)+m^{2}(P \psi)=0
$$

This shows that all elements of the column matrix $P \psi$ are scalar fields of mass $m$ obeying KG equation with minimal coupling, while the elements of $P^{\mu} \psi$ are $\frac{i}{m}$ times the covariant derivatives of the corresponding elements of $P \psi$. Following exactly the same steps and using the same representation for the matrices $\beta^{\mu}$ used to obtain expression (46) in the free field case, we see that the correct physical form for $\psi$ when we have electromagnetic interaction is given by equation (54). Besides that, we have

$$
P \psi=\left(\begin{array}{c}
0_{4 \times 1} \\
\sqrt{m} \varphi
\end{array}\right), P^{\mu} \psi=\frac{i}{\sqrt{m}}\left(\begin{array}{c}
0_{4 \times 1} \\
D^{\mu} \varphi
\end{array}\right), D^{\mu} D_{\mu} \varphi+m^{2} \varphi=0 .
$$

Furthermore, to regain KG interaction Lagrangian it is not enough to use the expression (54) for $\psi$ in the DKP interaction term (50), we must consider the whole DKP Lagrangian since now $\psi$ has covariant derivatives in its components and, consequently, "spread" the interaction terms throughout the whole DKP Lagrangian. So, we must substitute expression (54) in the complete minimally coupled DKP Lagrangian given by expression (55) or (56). This results in $\mathcal{L}=-\frac{1}{2}\left(\varphi^{*} \partial^{\mu} D_{\mu} \varphi+\varphi \partial^{\mu} D_{\mu}^{*} \varphi^{*}\right)+i e \frac{1}{2} A^{\mu}\left(\varphi^{*} \partial_{\mu} \varphi-\varphi \partial_{\mu} \varphi^{*}\right)+e^{2} A^{\mu} A_{\mu} \varphi \varphi^{*}-m^{2} \varphi^{*} \varphi$, 


$$
\begin{gathered}
\mathcal{L}=\frac{1}{2}\left(\partial^{\mu} \varphi^{*} D_{\mu} \varphi+\partial^{\mu} \varphi D_{\mu}^{*} \varphi^{*}\right)+i e \frac{1}{2} A^{\mu}\left(\varphi^{*} \partial_{\mu} \varphi-\varphi \partial_{\mu} \varphi^{*}\right)+e^{2} A^{\mu} A_{\mu} \varphi \varphi^{*}-m^{2} \varphi^{*} \varphi \\
\mathcal{L}=\partial^{\mu} \varphi^{*} \partial_{\mu} \varphi+i e A^{\mu}\left(\varphi^{*} \partial_{\mu} \varphi-\varphi \partial_{\mu} \varphi^{*}\right)+e^{2} A^{\mu} A_{\mu} \varphi \varphi^{*}-m^{2} \varphi^{*} \varphi,
\end{gathered}
$$

where an integration by parts was performed in the first term of equation (62). This is exactly the usual KG Lagrangian with minimal coupling so that we obtain the correct interaction term

$$
\mathcal{L}_{I}=i e A^{\mu}\left(\varphi^{*} \partial_{\mu} \varphi-\varphi \partial_{\mu} \varphi^{*}\right)+e^{2} A^{\mu} A_{\mu} \varphi \varphi^{*}
$$

Thus we see that, using the correct physical form for the DKP field $\psi$ in the minimal coupling case, we can recover the KG Lagrangian with the correct minimal coupling interaction term. Consequently, the equivalence of these theories is kept when electromagnetic interaction comes to play. The apparent difference reported in literature comes from the use of a physical form for the DKP field, obtained in the free case, that is no longer valid with the presence of the electromagnetic field and is incompatible with the requirement of local gauge invariance.

\section{The physical components and the anomalous terms}

Now we will analyse the presence of an apparently anomalous term lacking physical interpretation in Hamiltonian and second order forms of minimally coupled DKP theory. These forms are obtained starting from the DKP equation with minimal coupling, equation (57), and contracting from the left with $D_{\alpha} \beta^{\alpha} \beta^{\nu}$, which results in

$$
i \beta^{\alpha} \beta^{\nu} \beta^{\mu} D_{\alpha} D_{\mu} \psi-m D_{\alpha} \beta^{\alpha} \beta^{\nu} \psi=0 .
$$


After some algebraic calculation the above expression reduces to

$$
D^{\nu} \psi=\beta^{\mu} \beta^{\nu} D_{\mu} \psi+\frac{e}{2 m} F_{\alpha \mu}\left(\beta^{\mu} \beta^{\nu} \beta^{\alpha}+\beta^{\mu} \eta^{\nu \alpha}\right) \psi,
$$

where the relation $\left[D_{\mu}, D_{\nu}\right]=-i e F_{\mu \nu}$ was used. Making $\nu=0$ in this last expression we have

$$
D^{0} \psi-\left(\beta^{0}\right)^{2} D_{0} \psi-\beta^{i} \beta^{0} D_{i} \psi-\frac{e}{2 m} F_{\alpha \mu}\left(\beta^{\mu} \beta^{0} \beta^{\alpha}+\beta^{\mu} \eta^{0 \alpha}\right) \psi=0,
$$

while multiplying equation (57) from left by $-i \beta^{0}$ results in

$$
\left(\beta^{0}\right)^{2} D_{0} \psi+\beta^{0} \beta^{i} D_{i} \psi+i m \beta^{0} \psi=0
$$

Then, summing these equations we get the Hamiltonian (Schrödinger like) form of DKP wave equation

$$
i \partial_{t} \psi=H \psi
$$

where

$$
H=i\left[\beta^{i}, \beta^{0}\right] D_{i}+i \frac{e}{2 m} F_{\alpha \mu}\left(\beta^{\mu} \beta^{0} \beta^{\alpha}+\beta^{\mu} \eta^{0 \alpha}\right)-e A^{0}+m \beta^{0} .
$$

Finally, contracting equation (67) with $D_{\nu}$ we get as result a second order wave equation

$$
D_{\nu} D^{\nu} \psi+m^{2} \psi-\frac{i}{2} e F_{\mu \nu} S^{\mu \nu} \psi-\frac{e}{2 m}\left(\beta^{\mu} \beta^{\nu} \beta^{\alpha}+\beta^{\mu} \eta^{\nu \alpha}\right) D_{\nu}\left(F_{\alpha \mu} \psi\right)=0 .
$$

The anomalous term is the one proportional to $e / 2 m$ in equations (67) and (72) and in Hamiltonian (71). This denomination is due to the fact, already noticed by Kemmer in his original work [2], that it has no apparent physical interpretation, contrary to the others terms in these equations, which have physical interpretations analogous to similar terms obtained when working with Dirac equation. 
Recently Nowakowski [11] settled the question by showing that the above mentioned term has no physical meaning and simply disapears when one works with the physical components of the DKP fieldi'. This is done by the use a specific choice of the $10 \times 10$ and $5 \times 5$ representations of DKP algebra. Our intention here is to show that this result can be easily obtained in any representation of the algebra (it is not even necessary to be an irreducible one) through the use of the operators, defined in Section 2, that select the spin 0 and spin 1 sectors of the theory from any choice of the $\beta$ matrices satisfying DKP algebra.

First, for the case of the spin 0 sector of the theory, we can apply the operator $P$ defined by equation (17) to the second order equation (72) obtaining

$$
\begin{gathered}
D_{\nu} D^{\nu}(P \psi)+m^{2}(P \psi)-\frac{i}{2} e F_{\mu \nu} P S^{\mu \nu} \psi-\frac{e}{2 m} P\left(\beta^{\mu} \beta^{\nu} \beta^{\alpha}+\beta^{\mu} \eta^{\nu \alpha}\right) D_{\nu}\left(F_{\alpha \mu} \psi\right)=0 \\
D_{\nu} D^{\nu}(P \psi)+m^{2}(P \psi)-\frac{e}{2 m}\left(\eta^{\mu \nu} P^{\alpha}+P^{\mu} \eta^{\nu \alpha}\right) D_{\nu}\left(F_{\alpha \mu} \psi\right)=0 \\
D_{\nu} D^{\nu}(P \psi)+m^{2}(P \psi)=0,
\end{gathered}
$$

where we have used equations (18) and (19). So we see that when we select the spin 0 sector $P \psi$ the anomalous term vanishes. More than this, the spin-field interaction term [2] $F_{\mu \nu} S^{\mu \nu}$ also vanishes, as it should be for a scalar field. When applying $P^{\lambda}$ to this equation we get as result the covariant derivative $D^{\lambda}$ of $\mathrm{KG}$ equation (75), but we must remember that the relations between $P$ and $P^{\lambda}$ are now given by equations (58) and (59) and not by equations (22) and (23) obtained for the free field case. Analogously, when using $P$ and $P^{\lambda}$ on equations (67) and (70) we obtain trivial equalities or regain KG equation (75).

\footnotetext{
${ }^{\dagger}$ Moreover, he shows that the solutions of the second order equation (72) will not always be solutions of the first order DKP equation. So, it is not a good analog to Dirac's second order equation. He also shows that (72) is just one among a class of second order equations that can be obtained from DKP equation.
} 
Now, in order to analyse the spin 1 sector, we must derive the relation between $R^{\mu} \psi$ and $R^{\mu \nu} \psi$ in the presence of minimal coupling, in the same way that we have done in the previous section with the operators $P$ and $P^{\mu}$. So, we apply those operators to the DKP equation with minimal coupling, equation (57), obtaining

$$
D_{\alpha}\left(R^{\mu \alpha} \psi\right)=\frac{m}{i}\left(R^{\mu} \psi\right),
$$

and

$$
\left(R^{\mu \nu} \psi\right)=-\frac{i}{m} U^{I \mu \nu},
$$

where

$$
U^{I \mu \nu}=D^{\mu} R^{\nu} \psi-D^{\nu} R^{\mu} \psi
$$

is the covariant stress tensor of the massive vector field $R^{\mu} \psi$ interacting minimally with electromagnetic field. This tensor can be written explicitly as

$$
U^{I \mu \nu}=U^{\mu \nu}-i e\left(A^{\mu} R^{\nu} \psi-A^{\nu} R^{\mu} \psi\right) .
$$

It is interesting to mention that combining these results we have the minimally coupled Proca equation

$$
D_{\alpha}\left(U^{I \alpha \mu}\right)+m^{2}\left(R^{\mu} \psi\right)=0 .
$$

Now, when we apply the operator $R^{\lambda}$ to equation (72) we get

$$
\begin{aligned}
D_{\nu} D^{\nu} R^{\lambda} \psi+m^{2} R^{\lambda} \psi & -\frac{i}{2} e F_{\mu \nu} R^{\lambda} S^{\mu \nu} \psi \\
& -\frac{e}{2 m}\left(R^{\lambda \mu} \beta^{\nu} \beta^{\alpha}+R^{\lambda \mu} \eta^{\nu \alpha}\right) D_{\nu}\left(F_{\alpha \mu} \psi\right)=0,
\end{aligned}
$$

which can be shown to reduce, after some algebraic manipulation, to the minimally coupled Proca equation (80). When we apply the operator $R^{\lambda \alpha}$ to the second order equation (72) we simply regain the $U^{I \mu \nu}$ definition, equation (79). 
Similar results follow to the others equations: we regain definitions or get trivial identities. As example, applying $R^{\lambda}$ to equation (67) will result in

$$
D_{\mu} R^{\mu} \psi=\frac{i e}{2 m^{2}} F_{\alpha \mu} U^{I \mu \alpha}
$$

where $F_{\alpha \mu}$ is the usual stress tensor for electromagnetic field $A_{\mu}$. But this result can be obtained directly from the covariant derivative of Proca equation (80).

The important result is that when we select the physical components of DKP field $\psi$ the anomalous term is eliminated, so it has no physical meaning. And, as mentioned before, this result is not dependent upon a specific choice of the $\beta$ matrices nor the degree of the representation, being quite general.

\section{Conclusions and comments}

In this work we discussed two points relative to the minimal electromagnetic coupling in DKP theory that is not usually understood in literature correctly.

First we showed that a reported difference between the interaction term for scalar bosons in spin 0 DKP theory and KG theory does not exist. This apparent difference was showed to be caused by the incorrect use of an expression for the physical form of DKP field that is valid only in the free field case, not in the

minimally coupled case. When the correct physical form of $\psi$ in the presence of electromagnetic interaction is used, we find no difference between the interaction terms in both theories. We have also shown that the gauge invariance principle shows clearly the origin of the problem since the free field physical form of $\psi$ is incompatible with gauge invariance: changing the expression for physical form is necessary to keep gauge invariance. Moreover, the correct expression was easily obtained using DKP Lagrangian with minimal coupling, the minimally coupled DKP equation, the projectors of the physical components and a convenient choice 
of $\beta$ matrices (the same used in the free field case).

In addition we showed that the apparently anomalous term in second order and Hamiltonian forms of DKP equation disappears when we select the physical components of DKP field, so that this term has no physical meaning. This conclusion is exactly the same presented by Nowakowski [11] but, it seems to us, was obtained here in a more clear and becoming way. Furthermore, the use of the operators that select the spin 0 and 1 sectors of the theory do not demand the choice of an specific representation of the $\beta$ matrices. Finally, it is also interesting to notice that, when we project the physical components of DKP field, the second order equation (72) is reduced to the well known second order equations for scalar and vector fields, i.e. Klein-Gordon and Proca equations.

\section{Acknowlegdments}

J.T.L. and B.M.P. would like to thank CAPES's PICDT program and CNPq, respectively, for partial support. R.G.T. and J.S.V. thank CAPES and FAPESP (grant 98/00268-1), respectively, for full support.

\section{References}

[1] R. J. Duffin, Phys. Rev. 54 (1938), 1114.

[2] N. Kemmer, Proc. Roy. Soc. A173 (1939), 91.

[3] G. Petiau, University of Paris thesis (1936). Published in Acad. Roy. de Belg., Classe Sci., Mem in $8^{\circ} 16$ (1936), No. 2.

[4] R. A. Krajcik and M. M. Nieto, Am. J. Phys. 45 (1977), 818. 
[5] V. Gribov, Eur. Phys. J. C10:(1999), 71. Also available as hep-ph/9807224.

[6] I. V. Kanatchikov, hep-th/9911175.

[7] V. M. Red'kov, quant-ph/9812007.

[8] J. T. Lunardi, B. M. Pimentel and R. G. Teixeira, gr-qc/9909033.

[9] H. Umezawa, "Quantum Field Theory," North-Holland, 1956.

[10] A. I. Akhiezer and V. B. Berestetskii, "Quantum Electrodynamics," Interscience, 1965.

[11] M. Nowakowski, Phys. Lett. A244 (1998), 329.

[12] V. Ya. Fainberg and B. M. Pimentel, hep-th/9911219. 